\title{
The ASTERICS Virtual Observatory Schools: Getting closer to the astronomical community
}

\section{Enrique Solano}

Departamento de Astrofísica, Centro de Astrobiología (CSIC-INTA), ESAC Campus, Camino Bajo del Castillo s/n, E-28692, Villanueva de la Cañada, Madrid, Spain

E-mail: esmecab.inta-csic.es

\section{Ada Nebot Gómez-Morán ${ }^{1}$}

Observatoire Astronomique de Strasbourg,Université de Strasbourg, CNRS, UMR 7550, 11 rue de

l'Université, 67000, Strasbourg, France

E-mail: ada.nebot@astro.unistra.fr

\section{Francoise Genova}

CDS, Université de Strasbourg, CNRS, Observatoire astronomique de Strasbourg, UMR 7550

11 rue de l'Université, 67000 Strasbourg, France

E-mail: francoise.genova@astro.unistra.fr

The goal of ASTERICS WP4 (Data Access, Discovery and Interoperability) was to ensure that the ESFRI products become openly accessible via the Virtual Observatory framework to the international community. Training activities to efficiently use these new resources was identified as one of the important objectives within this work package. In this context, four Virtual Observatory (VO) schools were organised with a twofold objective:

- Expose European astronomers (in particular early-career people) to the variety of VO tools and services available today so that they can use them efficiently for their own research.

- $\quad$ Gather feedback and requirements from this intense and diverse usage.

During the schools, VO experts guided the participants on the usage of VO tools through a series of tutorials built based on real life science cases. Participants also had the opportunity to develop their own science cases.

One hundred and thirty-six participants from 16 different European countries attended the schools. Feedback gathered from participants demonstrated that the goals of the schools were fulfilled and that they were efficient steps forward in the process of building an astronomical VO community both within ASTERICS and beyond the project's borders across Europe.

The New Era of Multi-Messenger Astrophysics - Asterics2019

25 - 29 March, 2019

Groningen, The Netherlands

${ }^{1}$ Speaker 


\section{Introduction}

The European Virtual Observatory (Euro-VO) began to organise Virtual Observatory schools during the EuroVO-AIDA FP7 project (2008-2010). The usefulness of these schools was immediately obvious and they were continued in the framework of two coordination actions: EuroVO-ICE (2010-2012) and CoSADIE (2012-2015).

In the framework of the ASTERICS projects, VO schools were identified as an important line of work within work package \#4 ("Data Access, Discovery and Interoperability"). Schools were scheduled on a yearly basis (four schools in total) at two different venues: Centro de Astrobiología (CSIC-INTA, Madrid, Spain) and l'Observatoire Astronomique de Strasbourg (France).

\section{The Schools}

\subsection{Preparatory work}

Several months in advance, the school was widely advertised in the European community beyond ASTERICS through different channels (distribution list of national astronomical societies, ASTRONET, participants in previous schools,...). A school web site was set up in order to provide participants with all the necessary information before and during the meeting. Instructions on the software and its installation, as well as information on logistics were shared with the participants well in advance.

To take better advantage of the school, the students were encouraged to propose a scientific case related to their research. Tutors iterated with the students for several weeks, with the scope of better understanding the science case and to guide them before and during the school.

\subsection{The Programme}

The meeting programme had a similar structure for the four schools: introduction, tutorials, uses cases proposed by participants and feedback and wrap-up sessions.

Two introductory talks about the ASTERICS project, the school, the Virtual Observatory and the new lines of research that can be addressed with it ("VO Science") were presented during the first session. Given the different levels of knowledge on Virtual Observatory among the participants, we considered it necessary to have this type of presentations before starting with the practical cases.

The introduction session was followed by a hands-on session to make the participants familiar with the VO capabilities. Due to the heterogeneity in the knowledge of VO tools among the participants, it was decided to use tutorials of two different levels: beginners and intermediate. Tutorials were designed to cover the most important functionalities of the most popular VO tools. The last tutorial ("Treasure hunt") consisted in a series of challenging questions, to be answered in a limited time using the knowledge acquired with the previous 
tutorials. Three tutors were assigned to each tutorial. This ensured that the tutorial was carried out smoothly and within the allocated time, providing the participants with personalized support to solve questions that may arise during its execution.

The next session was devoted to the use cases provided by the students. During that session, students work together in small groups, use what they have learnt about the usage of the VO tools and services during the hands-on tutorials, and get help from the tutors.

The last session was allocated to discuss the answers provided by the participants to the questions proposed in the feedback form. This was an important session, as one of the main objectives of the school was to gather requirements from the community. The questionnaire, available on the website and filled on-line during the morning session of the last day, included questions about the knowledge of $\mathrm{VO}$ before the school, on each tutorial to evaluate difficulty and gather feedback for future schools, the plans to use VO-tools after the school and on different aspects of the organization and structure of the meeting.

\section{Conclusions}

The four schools were rated to be excellent/very good by the participants. ESFRI partners actively participated in the schools both as participants and as tutors (Figure 1).

A total of 136 participants from 16 different European countries attended the schools (Figure 2). The interactions with the participants before the school was time-consuming but rewarding given the successful outcome. This approach allowed the tutors to check the adequacy of the proposed projects with the $\mathrm{VO}$ beforehand and to identify the best methodology to conduct the project. Most of the participants submitted a science case.

The answers provided by participants in the feedback form served to identify new functionalities for the VO tools as well as to improve the organization and structure of the school itself.

The schools had a positive impact on the usage of VO tools to carry out astronomical research in Europe. Although most of the participants were early career researchers, 11 refereed papers and 7 contributions to conference proceedings (in both cases, most of them related to the science case proposed in the school), have been published by them since 2015 (see, for instance, Goldman et al. DOI:10.3847/1538-4357/aae64c).

The participants were encouraged to act as VO ambassadors in their research institutes by giving informal talks with colleagues, seminars and scientific workshops and conferences, and making use of the material employed during the school. Tutorials were "recycled" for similar initiatives at national level: 5 schools in Spain (one of them focused on Professional-Amateur activities), 2 in Poland, 1 in France and 1 in Italy benefited from using ASTERICS tutorials.

\section{Acknowledgements}

The authors acknowledge support from ASTERICS, a project funded by the European Commission under the Horizon2020 programme (id 653477), in the framework of ASTERICS Work Package 4 Data Access, Discovery and Interoperability. 


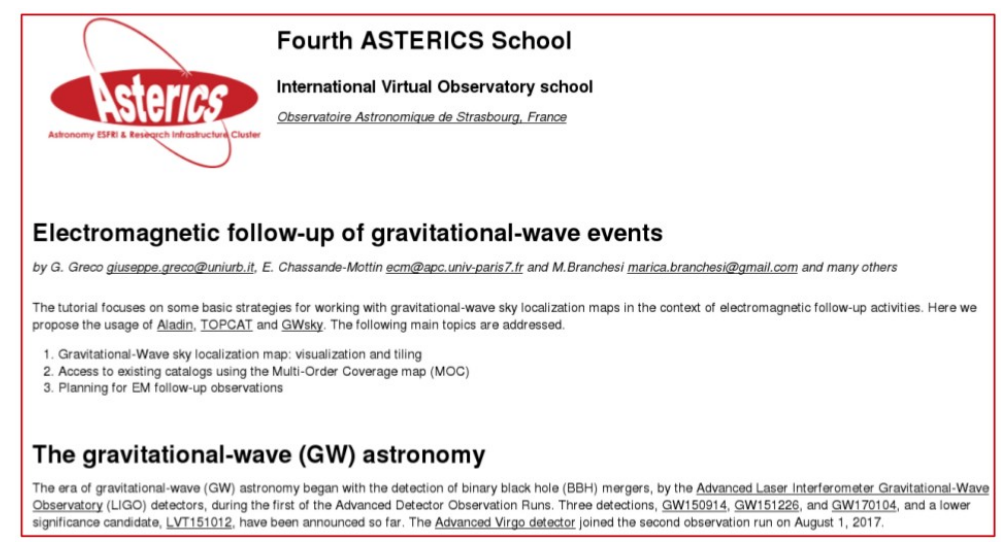

Figure 1. Tutorial prepared by EGO/VIRGO/ET on the follow-up of GW events using Virtual Observatory tools.

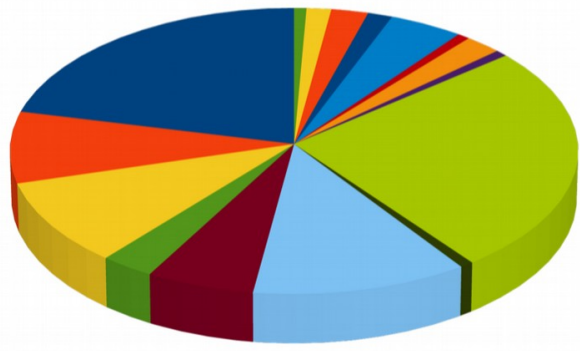

$\begin{array}{lll}\square \text { Spain (29) } & \text { United Kingdom (11) } & \text { Italy (13) } \\ \square \text { Hungary (4) } & \text { Germany (8) } & \text { Poland (16) } \\ \square \text { Austria (1) } & \text { France (35) } & \text { Belgium (1) } \\ \square \text { Greece (3) } & \text { Slovakia (1) } & \text { Sortugal (6) } \\ \square \text { The Netherlands (2) } & \text { Eithuania (3) } & \text { Bulgaria (2) } \\ \square \text { Ireland (1) } & & \end{array}$

Figure 2. Chart indicating the number of participants per country. 